\title{
Research Article \\ On the Causality between Multiple Locally Stationary Processes
}

\author{
Junichi Hirukawa \\ Faculty of Science, Niigata University, 8050 Ikarashi 2-no-cho, Nishi-ku, Niigata 950-2181, Japan \\ Correspondence should be addressed to Junichi Hirukawa, hirukawa@math.sc.niigata-u.ac.jp
}

Received 14 January 2012; Accepted 25 March 2012

Academic Editor: Kenichiro Tamaki

Copyright (C) 2012 Junichi Hirukawa. This is an open access article distributed under the Creative Commons Attribution License, which permits unrestricted use, distribution, and reproduction in any medium, provided the original work is properly cited.

When one would like to describe the relations between multivariate time series, the concepts of dependence and causality are of importance. These concepts also appear to be useful when one is describing the properties of an engineering or econometric model. Although the measures of dependence and causality under stationary assumption are well established, empirical studies show that these measures are not constant in time. Recently one of the most important classes of nonstationary processes has been formulated in a rigorous asymptotic framework by Dahlhaus in (1996), (1997), and (2000), called locally stationary processes. Locally stationary processes have time-varying spectral densities whose spectral structures smoothly change in time. Here, we generalize measures of linear dependence and causality to multiple locally stationary processes. We give the measures of linear dependence, linear causality from one series to the other, and instantaneous linear feedback, at time $t$ and frequency $\lambda$.

\section{Introduction}

In discussion of the relations between time series, concepts of dependence and causality are frequently invoked. Geweke [1] and Hosoya [2] have proposed measures of dependence and causality for multiple stationary processes (see also Taniguchi et al. [3]). They have also showed that these measures can be additively decomposed into frequency-wise. However, it seems to be restrictive that these measures are constants all the time. Priestley [4] has developed the extensions of prediction and filtering theory to nonstationary processes which have evolutionary spectra. Alternatively, in this paper we generalize measures of dependence and causality to multiple locally stationary processes.

When we deal with nonstationary processes, one of the difficult problems to solve is how to set up an adequate asymptotic theory. To meet this Dahlhaus [5-7] introduced an important class of nonstationary processes and developed the statistical inference. We give the precise definition of multivariate locally stationary processes which is due to Dahlhaus [8]. 
Definition 1.1. A sequence of multivariate stochastic processes $Z_{t, T}=\left(Z_{t, T}^{(1)}, \ldots, Z_{t, T}^{\left(d^{(Z)}\right)}\right)^{\prime},(t=$ $2-N / 2, \ldots, 1, \ldots, T, \ldots, T+N / 2 ; T, N \geq 1$ ) is called locally stationary with mean vector 0 and transfer function matrix $\mathrm{A}^{\circ}$ if there exists a representation

$$
\mathbf{Z}_{t, T}=\int_{-\pi}^{\pi} \exp (i \lambda t) \mathbf{A}_{t, T}^{\circ}(\lambda) d \zeta(\lambda)
$$

where

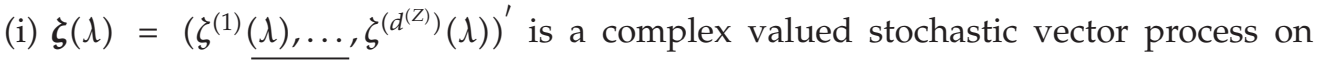
$[-\pi, \pi]$ with $\overline{\zeta^{(a)}(\lambda)}=\zeta^{(a)}(-\lambda)$ and

$$
\operatorname{cum}\left\{d \zeta^{\left(a_{1}\right)}\left(\lambda_{1}\right), \ldots, d \zeta^{\left(a_{k}\right)}\left(\lambda_{k}\right)\right\}=\eta\left(\sum_{j=1}^{k} \lambda_{j}\right) \frac{\kappa_{a_{1}, \ldots, a_{k}}}{(2 \pi)^{k-1}} d \lambda_{1} \cdots d \lambda_{k-1}
$$

for $k \geq 2, a_{1}, \ldots, a_{k}=1, \ldots, d^{(Z)}$, where cum $\{\cdots\}$ denotes the cumulant of $k$ th order, and $\eta(\lambda)=\sum_{j=-\infty}^{\infty} \delta(\lambda+2 \pi j)$ is the period $2 \pi$ extension of the Dirac delta function.

(ii) There exists a constant $K$ and a $2 \pi$-periodic matrix valued function $\mathbf{A}:[0,1] \times \mathbb{R} \rightarrow$ $\mathbb{C}^{d^{(Z)} \times d^{(Z)}}$ with $\mathbf{A}(u,-\lambda)=\overline{\mathbf{A}(u, \lambda)}$ and

$$
\sup _{t, \lambda}\left|\mathbf{A}_{t, T}^{\circ}(\lambda)_{a, b}-\mathbf{A}\left(\frac{t}{T}, \lambda\right)_{a, b}\right| \leq K T^{-1}
$$

for all $a, b=1, \ldots, d^{(Z)}$ and $T \in \mathbb{N}$. $\mathbf{A}(u, \lambda)$ is assumed to be continuous in $u$.

We call $\mathbf{f}(u, \lambda):=\mathbf{A}(u, \lambda) \boldsymbol{\Omega} \mathbf{A}(u, \lambda)^{*}$ the time-varying spectral density matrix of the process, where $\boldsymbol{\Omega}=\left(\kappa_{a, b}\right)_{a, b=1, \ldots, d^{(z)}}$. Write

$$
\boldsymbol{\epsilon}_{t}:=\int_{-\pi}^{\pi} \exp (i \lambda t) d \zeta(\lambda)
$$

then $\left\{\boldsymbol{\epsilon}_{t}\right\}$ becomes a white noise process with $E\left(\boldsymbol{\epsilon}_{t}\right)=\mathbf{0}$ and $\operatorname{Var}\left(\boldsymbol{\epsilon}_{t}\right)=\boldsymbol{\Omega}$.

Our objective is the generalization of dependence and causality measures to locally stationary processes and construction of test statistics which can examine the nonstationary effect of actual time series data. The paper, organized as follows. Section 2 explains the generalization of causality measures to multiple locally stationary processes. Since this extension is natural, we do is not explain the original idea of the causality measures in stationary case and recommend to refer Geweke [1] and Hosoya [2] for it. In Section 3 we introduce the nonparametric spectral estimator of multivariate locally stationary processes and explain their asymptotic properties. Finally, we propose the test statistics for linear dependence and show their performance in terms of empirical numerical example in Section 4 . 


\section{Measurements of Linear Dependence and Causality for Nonstationary Processes}

Here, we generalize measures of dependence and causality to multiple locally stationary processes. The assumptions and results of this section are straightforward extension of the original idea in stationary case. To avoid repetition, Geweke [1] and Hosoya [2] should be referred to for the original idea of causality.

For the $d^{(Z)}$-dimensional locally stationary process $\left\{\mathbf{Z}_{t, T}\right\}$, we introduce $\mathscr{H}$, the Hilbert space spanned by $Z_{t, T}^{(j)}, j=1, \ldots, d^{(Z)}, t=0, \pm 1, \ldots$, and call $\mathscr{H}\left(\mathbf{Z}_{t, T}\right)$ the closed subspace spanned by $Z_{s, T}^{(j)}, j=1, \ldots, d^{(Z)}, s \leq t$. We obtain the best one-step linear predictor of $\mathbf{Z}_{t+1, T}$ by projecting the components of the vector onto $\mathscr{H}\left(\mathbf{Z}_{t, T}\right)$, so here projection implies componentwise projection. We denote the error of prediction by $\xi_{t+1, T}$. Then, for locally stationary process we have

$$
E\left(\boldsymbol{\xi}_{s, T} \boldsymbol{\xi}_{t, T}^{\prime}\right)=\delta_{s, t} G_{t, T}
$$

where $\delta_{s, t}$ is the Kronecker delta function. Note that $\boldsymbol{\xi}_{t, T}{ }^{\prime} \mathrm{s}$ are uncorrelated but do not have identical covariance matrices; namely, $G_{t, T}$ are time-dependent. Now, we impose the following assumption on $G_{t, T}$.

Assumption 2.1. The covariance matrices of errors $G_{t, T}$ are nonsingular for all $t$ and $T$.

Define

$$
\mathbf{u}_{t, T}=\sum_{j=0}^{\infty} H_{t, T}(j) \boldsymbol{\xi}_{t-j, T}, \quad \operatorname{tr}\left\{\sum_{j=0}^{\infty} H_{t, T}(j) G_{t-j, T} H_{t, T}(j)^{\prime}\right\}<\infty
$$

as a one-sided linear process and

$$
\mathbf{v}_{t, T}=\mathbf{Z}_{t, T}-\mathbf{u}_{t, T}
$$

where coefficient matrices are

$$
H_{t, T}(j)=E\left(\mathbf{Z}_{t, T} \xi_{t-j, T}^{\prime}\right) G_{t, T}^{-1}, \quad j \geq 1, \quad H_{t, T}(0)=I_{d(z)}
$$

Note that each $H_{t, T}(j) \boldsymbol{\xi}_{t-j, T}, j=0,1, \ldots$ is projection of $\mathbf{Z}_{t, T}$ onto the closed subspace spanned by $\xi_{t-j, T}$. Now, we have the following Wold decomposition for locally stationary processes.

Lemma 2.2 (Wold decomposition). If $\left\{\mathbf{Z}_{t, T}\right\}$ is a locally stationary vector process of $d^{(Z)}$ components, then $\mathbf{Z}_{t, T}=\mathbf{u}_{t, T}+\mathbf{v}_{t, T}$, where $\mathbf{u}_{t, T}$ is given by (2.1), (2.2), and (2.4), $\mathbf{v}_{t, T}$ is deterministic, and $E\left(\mathbf{v}_{s, T} \boldsymbol{\xi}_{t, T}^{\prime}\right) \equiv 0$.

If only $\mathbf{u}_{t, T}$ occurs, we say that $\mathbf{Z}_{t, T}$ is purely nondeterministic.

Assumption 2.3. $\mathbf{Z}_{t, T}$ is purely nondeterministic. 
In view of Lemma 2.2, we can see that under Assumptions 2.1 and 2.3, $\mathbf{Z}_{t, T}$ becomes a one-side linear process given by (2.2). For locally stationary process, if we choose an orthonormal basis $\varepsilon_{t}^{(j)}, j=1, \ldots, d^{(Z)}$, in the closed subspace spanned by $\xi_{t, T}$, then $\left\{\varepsilon_{t}\right\}$ will be an uncorrelated stationary process. We call $\left\{\varepsilon_{t}\right\}$ a fundamental process of $\left\{\mathbf{Z}_{t, T}\right\}$ and $C_{t, T}(j)$; $j=0,1, \ldots$ denote the corresponding coefficients, that is,

$$
\mathbf{Z}_{t, T}=\sum_{j=0}^{\infty} C_{t, T}(j) \varepsilon_{t-j}
$$

Let $\mathbf{f}_{t, T}(\lambda)$ be the time-varying spectral density matrix of $\mathbf{Z}_{t, T}$. A process is said to have the maximal rank if it has nondegenerate spectral density matrix a.e.

Assumption 2.4. The locally stationary process $\left\{\mathbf{Z}_{t, T}\right\}$ has the maximal rank for all $t$ and $T$. In particular

$$
\int_{-\pi}^{\pi} \log \left|\mathbf{f}_{t, T}(\lambda)\right| d \lambda>-\infty, \quad \forall t, T
$$

where $|D|$ denotes the determinant of the matrix $D$.

We will say that a function $\phi(z)$, analytic in the unit disc, belongs to the class $\mathscr{d}_{2}$ if

$$
H_{2}(\phi)=\sup _{0 \leq \rho<1} \int_{-\pi}^{\pi}\left|\phi\left(\rho e^{-i \lambda}\right)\right|^{2} d \lambda<\infty
$$

Under Assumptions 2.1-2.4, it follows that $\left\{\mathbf{Z}_{t, T}\right\}$ has a time-varying spectral density $\mathbf{f}_{t, T}(\lambda)$ which has rank $d^{(Z)}$ for almost all $\lambda$, and is representable in the form

$$
\mathbf{f}_{t, T}(\lambda)=\frac{1}{2 \pi} \boldsymbol{\Phi}_{t, T}\left(e^{i \curlywedge}\right) \boldsymbol{\Phi}_{t, T}\left(e^{i \curlywedge}\right)^{*}
$$

where $\mathbf{D}^{*}$ denotes the complex conjugate of matrix $\mathbf{D}$ and $\boldsymbol{\Phi}_{t, T}\left(e^{i \lambda}\right)$ is the boundary value of a $d^{(Z)} \times d^{(Z)}$ analytic function

$$
\mathbf{\Phi}_{t, T}(z)=\sum_{j=0}^{\infty} C_{t, T}(j) z^{j}
$$

in the unit disc, and it holds that $\boldsymbol{\Phi}_{t, T}(0) \boldsymbol{\Phi}_{t, T}(0)^{*}=G_{t, T}$.

Now, we introduce measures of linear dependence, linear causality, and instantaneous linear feedback at time $t$. Let $\mathbf{Z}_{t, T}=\left(\mathbf{X}_{t, T}^{\prime}, \mathbf{Y}_{t, T}^{\prime}\right)^{\prime}$ be $d^{(Z)}=\left(d^{(X)}+d^{(Y)}\right)$-dimensional locally stationary process, which has time-varying spectral density matrix:

$$
\mathbf{f}_{t, T}(\lambda)=\left(\begin{array}{cc}
\mathbf{f}_{t, T}^{(x x)}(\lambda) & \mathbf{f}_{t, T}^{(x y)}(\lambda) \\
\mathbf{f}_{t, T}^{(y x)}(\lambda) & \mathbf{f}_{t, T}^{(y y)}(\lambda)
\end{array}\right) .
$$


We will find the partitions $\boldsymbol{\xi}_{t, T}\left(\begin{array}{c|c}\boldsymbol{\xi}_{t, T}^{(1)} & d^{(X)} \times 1 \\ \boldsymbol{\xi}_{t, T}^{(2)} & d^{(Y)} \times 1\end{array}\right)$ and

$$
\operatorname{Cov}\left(\boldsymbol{\xi}_{t, T}, \boldsymbol{\xi}_{t, T}\right)=G_{t, T}=\left(\begin{array}{cc}
G_{t, T}^{(1,1)} & G_{t, T}^{(1,2)} \\
G_{t, T}^{(2,1)} & G_{t, T}^{(2,2)}
\end{array}\right)
$$

useful. Meanwhile $G_{t, T}^{(X)}$ and $G_{t, T}^{(Y)}$ denote the covariance matrices of the one-step-ahead errors $\boldsymbol{\xi}_{t, T}^{(X)}$ and $\boldsymbol{\xi}_{t, T}^{(Y)}$ when $\boldsymbol{X}_{t, T}$ and $\mathbf{Y}_{t, T}$ are forecasts from their own pasts alone; namely, $\boldsymbol{\xi}_{t, T}^{(X)}$ and $\boldsymbol{\xi}_{t, T}^{(Y)}$ are the residuals of the projections of $\mathbf{X}_{t, T}$ and $\mathbf{Y}_{t, T}$ onto $\mathscr{\ell}\left(\mathbf{X}_{t-1, T}\right)$ and $\mathscr{\ell}\left(\mathbf{Y}_{t-1, T}\right)$, respectively.

We define the measures of linear dependence, linear causality from $\left\{\mathbf{Y}_{t, T}\right\}$ to $\left\{\mathbf{X}_{t, T}\right\}$, from $\left\{\mathbf{X}_{t, T}\right\}$ to $\left\{\mathbf{Y}_{t, T}\right\}$ and instantaneous linear feedback, at time $t$ as

$$
\begin{gathered}
M_{t, T}^{(X, Y)}=\log \frac{\left|G_{t, T}^{(X)}\right|\left|G_{t, T}^{(Y)}\right|}{\left|G_{t, T}\right|}, \\
M_{t, T}^{(Y \rightarrow X)}=\log \frac{\left|G_{t, T}^{(X)}\right|}{\left|G_{t, T}^{(1,1)}\right|}, \\
M_{t, T}^{(X \rightarrow Y)}=\log \frac{\left|G_{t, T}^{(Y)}\right|}{\left|G_{t, T}^{(2,2)}\right|}, \\
M_{t, T}^{(X \cdot Y)}=\log \frac{\left|G_{t, T}^{(1,1)}\right|\left|G_{t, T}^{(2,2)}\right|}{\left|G_{t, T}\right|},
\end{gathered}
$$

respectively; then we have

$$
M_{t, T}^{(X, Y)}=M_{t, T}^{(Y \rightarrow X)}+M_{t, T}^{(X \rightarrow Y)}+M_{t, T}^{(X \cdot Y)} .
$$

Next, we decompose measures of linear causality into frequency-wise. To define frequency-wise measures of causality, we introduce the following analytic facts.

Lemma 2.5. The analytic matrix $\mathbf{\Phi}_{t, T}(z)$ corresponding to a fundamental process $\left\{\boldsymbol{\varepsilon}_{t}\right\}$ (for $\left\{\mathbf{Z}_{t, T}\right\}$ ) is maximal among analytic matrices $\Psi_{t, T}(z)$ with components from the class $\mathscr{d}_{2}$, and satisfying the boundary condition (2.8); that is,

$$
\boldsymbol{\Phi}_{t, T}(0) \boldsymbol{\Phi}_{t, T}(0)^{*} \geq \boldsymbol{\Psi}_{t, T}(0) \boldsymbol{\Psi}_{t, T}(0)^{*}
$$

Although the following assumption is natural extension of Kolmogorov's formula in stationary case (see, e.g., [9]), it is not straightforward and unfortunately, so far, we cannot prove it from more simple assumption. We guess it requires another completely technical paper. 
Assumption 2.6 (Kolmogorov's formula). Under Assumptions 2.1-2.4, an analytic matrix $\boldsymbol{\Phi}_{t, T}(z)$ satisfying the boundary condition (2.8) will be maximal if and only if

$$
\left|\boldsymbol{\Phi}_{t, T}(0)\right|^{2}=\left|G_{t, T}\right|=\exp \frac{1}{2 \pi} \int_{-\pi}^{\pi} \log \left|2 \pi \mathbf{f}_{t, T}(\lambda)\right| d \lambda .
$$

Now we define the process $\left\{\boldsymbol{\eta}_{t, T}\right\}$ as

$$
\left(\begin{array}{c}
\boldsymbol{\eta}_{t, T}^{(1)} \\
\boldsymbol{\eta}_{t, T}^{(2)}
\end{array}\right)=\left(\begin{array}{cc}
I_{d^{(X)}} & -G_{t, T}^{(1,2)} G_{t, T}^{(2,2)^{-1}} \\
-G_{t, T}^{(2,1)} G_{t, T}^{(1,1)^{-1}} & I_{d^{(Y)}}
\end{array}\right)\left(\begin{array}{c}
\xi_{t, T}^{(1)} \\
\xi_{t, T}^{(2)}
\end{array}\right),
$$

then $\boldsymbol{\eta}_{t, T}^{(1)}$ is the residuals of the projection of $\mathbf{X}_{t, T}$ onto $\mathscr{H}\left(\mathbf{X}_{t-1, T}, \mathbf{Y}_{t, T}\right)$, whereas $\boldsymbol{\eta}_{t, T}^{(2)}$ is the residuals of the projection of $\mathbf{Y}_{t, T}$ onto $\mathscr{H}\left(\mathbf{X}_{t, T}, \mathbf{Y}_{t-1, T}\right)$.

Furthermore, we have

$$
\begin{aligned}
\operatorname{Cov}\left\{\left(\begin{array}{c}
\boldsymbol{\xi}_{t, T}^{(1)} \\
\boldsymbol{\eta}_{t, T}^{(2)}
\end{array}\right),\left(\begin{array}{c}
\boldsymbol{\xi}_{t, T}^{(1)} \\
\boldsymbol{\eta}_{t, T}^{(2)}
\end{array}\right)\right\} & =\left(\begin{array}{cc}
G_{t, T}^{(1,1)} & 0 \\
0 & G_{t, T}^{(2,2)}-G_{t, T}^{(2,1)} G_{t, T}^{(1,1)} G_{t, T}^{(1,2)}
\end{array}\right) \\
& =\left(\begin{array}{cc}
G_{t, T}^{(1,1)} & 0 \\
0 & \tilde{G}_{t, T}^{(2,2)}
\end{array}\right)=\widetilde{G}_{t, T}=\widetilde{G}_{t, T}^{1 / 2} \widetilde{G}_{t, T}^{1 / 2}
\end{aligned}
$$

so we can see that $\boldsymbol{\eta}_{t, T}^{(2)}$ is orthogonal to $\boldsymbol{\xi}_{t, T}^{(1)}$. For a $d^{(Z)} \times d^{(Z)}$ matrix

$$
F_{t, T}=\left[\begin{array}{cc}
I_{d^{(X)}} & 0 \\
-G_{t, T}^{(2,1)} G_{t, T}^{(1,1)^{-1}} & I_{d^{(Y)}}
\end{array}\right],
$$

we have $\left(\begin{array}{c}\xi_{t, T}^{(1)} \\ \eta_{t, T}^{(2)}\end{array}\right)=F_{t, T}\left(\begin{array}{c}\xi_{t, T}^{(1)} \\ \xi_{t, T}^{(2)}\end{array}\right)$.

If we set

$$
\begin{aligned}
\tilde{\boldsymbol{\Phi}}_{t, T}(z) & =\boldsymbol{\Phi}_{t, T}(z) \boldsymbol{\Phi}_{t, T}(0)^{-1} F_{t, T}{ }^{-1} \tilde{G}_{t, T}^{1 / 2} \\
& =\boldsymbol{\Gamma}_{t, T}(z) \tilde{G}_{t, T}^{1 / 2},
\end{aligned}
$$

we have the following lemma.

Lemma 2.7. $\widetilde{\Phi}_{t, T}(z)$ is an analytic function in the unit disc with $\widetilde{\Phi}_{t, T}(0) \widetilde{\Phi}_{t, T}(0)^{*}=G_{t, T}$ and thus maximal, such that the time-varying spectral density $\mathbf{f}_{t, T}(\lambda)$ has a factorization

$$
\mathbf{f}_{t, T}(\lambda)=\frac{1}{2 \pi} \widetilde{\Phi}_{t, T}\left(e^{i \lambda}\right) \tilde{\Phi}_{t, T}\left(e^{i \lambda}\right)^{*} .
$$


From this lemma, it is seen that time-varying spectral density is decomposed into two parts:

$$
\mathbf{f}_{t, T}^{(x x)}(\lambda)=\frac{1}{2 \pi}\left\{\boldsymbol{\Gamma}_{t, T}^{(1,1)}\left(e^{i \curlywedge}\right) G_{t, T}^{(1,1)} \boldsymbol{\Gamma}_{t, T}^{(1,1)}\left(e^{i \curlywedge}\right)^{*}+\Gamma_{t, T}^{(1,2)}\left(e^{i \curlywedge}\right) \tilde{G}_{t, T}^{(2,2)} \boldsymbol{\Gamma}_{t, T}^{(1,2)}\left(e^{i \curlywedge}\right)^{*}\right\}
$$

where $\Gamma_{t, T}^{(1,1)}(z)$ is a $d^{(X)} \times d^{(X)}$ left-upper submatrix of $\Gamma_{t, T}(z)$. The former part is related to the process $\left\{\boldsymbol{\xi}_{t, T}^{(1)}\right\}$ whereas the latter part is related to the process $\left\{\boldsymbol{\eta}_{t, T}^{(2)}\right\}$, which is orthogonal to $\left\{\boldsymbol{\xi}_{t, T}^{(1)}\right\}$. This relation suggests frequency-wise measure of causality, from $\left\{\mathbf{Y}_{t, T}\right\}$ to $\left\{\mathbf{X}_{t, T}\right\}$ at time $t$ :

$$
M_{t, T}^{(Y \rightarrow X)}(\lambda)=\log \frac{\left|\mathbf{f}_{t, T}^{(x x)}(\lambda)\right|}{\left|(1 / 2 \pi)\left\{\Gamma_{t, T}^{(1,1)}\left(e^{i \lambda}\right) G_{t, T}^{(1,1)} \Gamma_{t, T}^{(1,1)}\left(e^{i \lambda}\right)^{*}\right\}\right|}
$$

Similarly, we propose

$$
\begin{gathered}
M_{t, T}^{(X \rightarrow Y)}(\lambda)=\log \frac{\left|\mathbf{f}_{t, T}^{(y y)}(\lambda)\right|}{\left|(1 / 2 \pi)\left\{\Delta_{t, T}^{(2,2)}\left(e^{i \lambda}\right) G_{t, T}^{(2,2)} \Delta_{t, T}^{(2,2)}\left(e^{i \lambda}\right)^{*}\right\}\right|}, \\
M_{t, T}^{(X, Y)}(\lambda)=-\log \mid I_{d^{(Y)}-\mathbf{f}_{t, T}^{(y x)}(\lambda) \mathbf{f}_{t, T}^{(x x)}(\lambda)^{-1} \mathbf{f}_{t, T}^{(x y)}(\lambda) \mathbf{f}_{t, T}^{(y y)}(\lambda)^{-1} \mid,} \\
M_{t, T}^{(X \cdot Y)}(\lambda)=\log \frac{\left|(1 / 2 \pi)\left\{\Gamma_{t, T}^{(1,1)}\left(e^{i \lambda}\right) G_{t, T}^{(1,1)} \Gamma_{t, T}^{(1,1)}\left(e^{i \lambda}\right)^{*}\right\}\right|\left|(1 / 2 \pi)\left\{\Delta_{t, T}^{(2,2)}\left(e^{i \lambda}\right) G_{t, T}^{(2,2)} \Delta_{t, T}^{(2,2)}\left(e^{i \lambda}\right)^{*}\right\}\right|}{\left|\mathbf{f}_{t, T}(\lambda)\right|},
\end{gathered}
$$

where $\Delta_{t, T}^{(2,2)}(z)$ is in the same manner of $\Gamma_{t, T}^{(1,1)}(z)$.

Now, we introduce the following assumption.

Assumption 2.8. The roots of $\left|\boldsymbol{\Gamma}_{t, T}^{(1,1)}(z)\right|$ and $\left|\boldsymbol{\Delta}_{t, T}^{(2,2)}(z)\right|$ all lie outside the unit circle. ing result.

The relation of frequency-wise measure to overall measure is addressed in the follow-

Theorem 2.9. Under Assumptions 2.1-2.8, we have

$$
M_{t, T}^{(\cdot)}=\frac{1}{2 \pi} \int_{-\pi}^{\pi} M_{t, T}^{(\cdot)}(\lambda) d \lambda
$$

If Assumptions 2.1-2.6 hold, but Assumption 2.8 does not hold, then

$$
M_{t, T}^{(Y \rightarrow X)}>\frac{1}{2 \pi} \int_{-\pi}^{\pi} M_{t, T}^{(Y \rightarrow X)}(\lambda) d \lambda, \quad M_{t, T}^{(X \rightarrow Y)}>\frac{1}{2 \pi} \int_{-\pi}^{\pi} M_{t, T}^{(X \rightarrow Y)}(\lambda) d \lambda .
$$


Remark 2.10. Since $\mathscr{H}\left(\mathbf{Z}_{t, T}\right)=\mathscr{H}\left(\boldsymbol{\xi}_{t, T}\right)=\mathscr{H}\left(\boldsymbol{\eta}_{t, T}\right)$ and $\mathscr{H}\left(\mathbf{Z}_{t, T}\right) \supseteq \mathscr{H}\left(\mathbf{X}_{t, T}, \boldsymbol{\eta}_{t, T}^{(2)}\right) \supseteq \mathscr{H}\left(\boldsymbol{\eta}_{t, T}\right)$, we can see that $\mathscr{H}\left(\mathbf{Z}_{t, T}\right)=\mathscr{H}\left(\mathbf{X}_{t, T}, \boldsymbol{\eta}_{t, T}^{(2)}\right)$. Therefore, the best one-step prediction error of the process $\left\{\begin{array}{l}x_{t, T} \\ \eta_{t, T}^{(2)}\end{array}\right\}$ is given by $\left\{\begin{array}{l}\xi_{t, T}^{(1)} \\ \eta_{t, T}^{(2)}\end{array}\right\}$. Let $\widetilde{\mathbf{f}}_{t, T}(\lambda)$ be a time-varying spectral density matrix of the process $\left\{\begin{array}{l}X_{t, T} \\ \eta_{t, T}^{(2)}\end{array}\right\}$ and denote the partition by

$$
\tilde{\mathbf{f}}_{t, T}(\lambda)=\left(\begin{array}{ll}
\mathbf{f}_{t, T}^{(x x)}(\lambda) & \tilde{\mathbf{f}}_{t, T}^{(1,2)}(\lambda) \\
\tilde{\mathbf{f}}_{t, T}^{(2,1)}(\lambda) & \frac{1}{2 \pi} \tilde{G}_{t, T}^{(2,2)}
\end{array}\right)
$$

Then, we obtain another representation of frequency-wise measure of causality, from $\left\{\mathbf{Y}_{t, T}\right\}$ to $\left\{\mathbf{X}_{t, T}\right\}$ at time $t$ :

$$
M_{t, T}^{(Y \rightarrow X)}(\lambda)=\log \frac{\left|\mathbf{f}_{t, T}^{(x x)}(\lambda)\right|}{\left|\mathbf{f}_{t, T}^{(x x)}(\lambda)-2 \pi \tilde{\mathbf{f}}_{t, T}^{(1,2)}(\lambda) \tilde{G}_{t, T}^{(2,2)_{-1}} \tilde{\mathbf{f}}_{t, T}^{(2,1)}(\lambda)\right|} .
$$

This relation suggests that we apply the nonparametric time-varying spectral density estimator of the residual process $\left\{\begin{array}{l}x_{t, T} \\ \widehat{\eta}_{t, T}^{(2)}\end{array}\right\}$. However, this problem requires another paper. We will make it as a further work.

\section{Nonparametric Spectral Estimator of Multivariate Locally Stationary Processes}

In this section we introduce the nonparametric spectral estimator of multivariate locally stationary processes. First, we make the following assumption on the transfer function matrix $\mathbf{A}(u, \lambda)$.

Assumption 3.1. (i) The transfer function matrix $\mathbf{A}(u, \lambda)$ is $2 \pi$-periodic in $\lambda$, and the periodic extension is twice differentiable in $u$ and $\lambda$ with uniformly bounded continuous derivatives $\partial^{2} / \partial u^{2} \mathbf{A}, \partial^{2} / \partial \lambda^{2} \mathbf{A}$ and $(\partial / \partial u)(\partial / \partial \lambda) \mathbf{A}$. Furthermore, the uniformly bounded continuous derivative $\left(\partial^{2} / \partial u^{2}\right)(\partial / \partial \lambda) \mathbf{A}$ also exists.

(ii) All the eigenvalues of $\mathbf{f}(u, \lambda)$ are bounded from below and above by some constants $\delta_{1}, \delta_{2}>0$ uniformly in $u$ and $\lambda$.

As an estimator of $\mathbf{f}(u, \lambda)$, we use the nonparametric estimator of kernel type defined by

$$
\widehat{\mathbf{f}}(u, \lambda)=\int_{-\pi}^{\pi} W_{T}(\lambda-\mu) \mathbf{I}_{N}(u, \mu) d \mu,
$$


where $W_{T}(\omega)=M \sum_{v=-\infty}^{\infty} W(M(\omega+2 \pi v))$ is the weight function and $M>0$ depends on $T$, and $\mathbf{I}_{N}(u, \lambda)$ is the localized periodogram matrix over the segment $\{[u T]-N / 2+1,[u T]+N / 2\}$ defined as

$$
\begin{aligned}
\mathbf{I}_{N}(u, \lambda)= & \frac{1}{2 \pi H_{2, N}}\left\{\sum_{s=1}^{N} h\left(\frac{s}{N}\right) \mathbf{Z}_{[u T]-N / 2+s, T} \exp \{i \lambda s\}\right\} \\
& \times\left\{\sum_{r=1}^{N} h\left(\frac{r}{N}\right) \mathbf{Z}_{[u T]-N / 2+r, T} \exp \{i \lambda r\}\right\}^{*} .
\end{aligned}
$$

Here $h:[0,1] \rightarrow \mathbb{R}$ is a data taper and $H_{2, N}=\sum_{s=1}^{N} h(s / N)^{2}$. It should be noted that $\mathbf{I}_{N}(u, \lambda)$ is not a consistent estimator of the time-varying spectral density. To make a consistent estimator of $\mathbf{f}(u, \lambda)$ we have to smooth it over neighbouring frequencies.

Now we impose the following assumptions on $W(\cdot)$ and $h(\cdot)$.

Assumption 3.2. The weighted function $W: \mathbb{R} \rightarrow[0, \infty]$ satisfies $W(x)=0$ for $x \notin[-1 / 2,1 / 2]$ and is continuous and even function satisfying $\int_{-1 / 2}^{1 / 2} W(x) d x=1$ and $\int_{-1 / 2}^{1 / 2} x^{2} W(x) d x<\infty$.

Assumption 3.3. The data taper $h: \mathbb{R} \rightarrow \mathbb{R}$ satisfies (i) $h(x)=0$ for all $x \notin[0,1]$ and $h(x)=$ $h(1-x)$; (ii) $h(x)$ is continuous on $\mathbb{R}$, twice differentiable at all $x \notin U$ where $U$ is a finite set of $\mathbb{R}$, and $\sup _{x \notin U}\left|h^{\prime \prime}(x)\right|<\infty$. Write

$$
K_{t}(x):=\left\{\int_{0}^{1} h(x)^{2} d x\right\}^{-1} h\left(x+\frac{1}{2}\right)^{2}, \quad x \in\left[-\frac{1}{2}, \frac{1}{2}\right]
$$

which plays a role of kernel in the time domain.

Furthermore, we assume the following.

Assumption 3.4. $M=M(T)$ and $N=N(T), M \ll N \ll T$ satisfy

$$
\frac{\sqrt{T}}{M^{2}}=o(1), \quad \frac{N^{2}}{T^{3 / 2}}=o(1), \quad \frac{\sqrt{T} \log N}{N}=o(1)
$$

The following lemmas are multivariate version of Theorem 2.2 of Dahlhaus [10] and Theorem A.2 of Dahlhaus [7] (see also [11]).

Lemma 3.5. Assume that Assumptions 3.1-3.4 hold. Then

(i)

$$
\begin{aligned}
E\left(\mathbf{I}_{N}(u, \lambda)\right)= & \mathbf{f}(u, \lambda)+\frac{N^{2}}{2 T^{2}} \int_{-1 / 2}^{1 / 2} x^{2} K_{t}(x)^{2} d x \frac{\partial^{2}}{\partial u^{2}} \mathbf{f}(u, \lambda) \\
& +o\left(\frac{N^{2}}{T^{2}}\right)+O\left(\frac{\log N}{N}\right),
\end{aligned}
$$


(ii)

$$
\begin{aligned}
E(\widehat{\mathbf{f}}(u, \lambda))= & \mathbf{f}(u, \lambda)+\frac{N^{2}}{2 T^{2}} \int_{-1 / 2}^{1 / 2} x^{2} K_{t}(x)^{2} d x \frac{\partial^{2}}{\partial u^{2}} \mathbf{f}(u, \lambda) \\
& +\frac{1}{2 M^{2}} \int_{-1 / 2}^{1 / 2} x^{2} W(x)^{2} d x \frac{\partial^{2}}{\partial \lambda^{2}} \mathbf{f}(u, \lambda) \\
& +o\left(\frac{N^{2}}{T^{2}}+M^{-2}\right)+O\left(\frac{\log N}{N}\right)
\end{aligned}
$$

(iii)

$$
\begin{aligned}
\sum_{i, j=1}^{m} \operatorname{Var}\left(\widehat{f}_{i, j}(u, \lambda)\right)= & \frac{M}{N} \sum_{i, j=1}^{m} f_{i, j}(u, \lambda)^{2} \int_{-1 / 2}^{1 / 2} K_{t}(x)^{2} d x \\
& \times \int_{-1 / 2}^{1 / 2} W(x)^{2} d x(2 \pi+2 \pi\{\lambda \equiv 0 \bmod \pi\})+o\left(\frac{M}{N}\right) .
\end{aligned}
$$

Hence, we have

$$
E\|\widehat{\mathbf{f}}(u, \lambda)-\mathbf{f}(u, \lambda)\|^{2}=O\left(\frac{M}{N}\right)+O\left(M^{-2}+N^{2} T^{-2}\right)^{2}=O\left(\frac{M}{N}\right),
$$

where $\|D\|$ is the Euclidean norm of the matrix $D$ and $\|D\|=\left\{\operatorname{tr}\left\{D D^{*}\right\}\right\}^{1 / 2}$.

Lemma 3.6. Assume that Assumptions 3.1-3.4 hold. Let $\phi_{j}(u, \lambda), j=1, \ldots, k$ be $d^{(Z)} \times d^{(Z)}$ matrixvalued continuous function on $[0,1] \times[-\pi, \pi]$ which satisfies the same conditions as the transfer function matrix $\mathbf{A}(u, \lambda)$ in Assumption 3.1 and $\phi_{j}(u, \lambda)^{*}=\phi_{j}(u, \lambda), \phi_{j}(u,-\lambda)=\phi_{j}(u, \lambda)^{\prime}$. Then

$$
\begin{aligned}
L_{T}\left(\phi_{j}\right)=\sqrt{T}\{ & \frac{1}{T} \sum_{t=1}^{T} \int_{-\pi}^{\pi} \operatorname{tr}\left\{\phi_{j}\left(\frac{t}{T}, \lambda\right) \mathbf{I}_{N}\left(\frac{t}{T}, \lambda\right)\right\} d \lambda \\
& \left.\quad-\int_{0}^{1} \int_{-\pi}^{\pi} \operatorname{tr}\left\{\phi_{j}(u, \lambda) \mathbf{f}(u, \lambda)\right\} d \lambda d u\right\}, \quad j=1, \ldots, k
\end{aligned}
$$


have, asymptotically, a normal distribution with zero mean vector and covariance matrix $V$ whose $(i, j)$-the element is

$$
\begin{aligned}
4 \pi \int_{0}^{1}\left[\int_{-\pi}^{\pi} \operatorname{tr}\left\{\phi_{i}(u, \lambda) \mathbf{f}(u, \lambda) \phi_{j}(u, \lambda) \mathbf{f}(u, \lambda)\right\} d \lambda\right. \\
\quad+\frac{1}{4 \pi^{2}} \sum_{a_{1}, a_{2}, a_{3}, a_{4}} \sum_{b_{1}, b_{2}, b_{3}, b_{4}} \kappa_{b_{1}, b_{2}, b_{3}, b_{4}} \\
\quad \times \int_{-\pi}^{\pi} \int_{-\pi}^{\pi} \phi_{i}(u, \lambda)_{a_{1}, a_{2}} \phi_{j}(u, \mu)_{a_{4}, a_{3}} \cdot \mathbf{A}(u, \lambda)_{a_{2}, b_{1}} \mathbf{A}(u,-\lambda)_{a_{1}, b_{2}} \\
\left.\quad \times \mathbf{A}(u,-\mu)_{a_{4}, b_{3}} \mathbf{A}(u, \mu)_{a_{3}, b_{4}} d \lambda d \mu\right] d u .
\end{aligned}
$$

Assumption 3.4 does not coincide with Assumption A.1(ii) of Dahlhaus [7]. As mentioned in A.3 Remarks of Dahlhaus [7, page 27], Assumption A.1(ii) of Dahlhaus [7] is required because of the $\sqrt{T}$-unbiasedness at the boundary 0 and 1 . If we assume that $\left\{\mathbf{Z}_{2-N / 2, T}, \ldots, \mathbf{Z}_{0, T}\right\}$ and $\left\{\mathbf{Z}_{T+1, T}, \ldots, \mathbf{Z}_{T+N / 2, T}\right\}$ are available with Assumption 3.4, then from Lemma 3.5 (i)

$$
\begin{aligned}
E\left(L_{T}\left(\phi_{j}\right)\right)= & \sqrt{T} E\left\{\frac{1}{T} \sum_{t=1}^{T} \int_{-\pi}^{\pi} \operatorname{tr}\left\{\phi_{j}\left(\frac{t}{T}, \lambda\right) \mathbf{I}_{N}\left(\frac{t}{T}, \lambda\right)\right\} d \lambda\right. \\
& \left.\quad-\int_{0}^{1} \int_{-\pi}^{\pi} \operatorname{tr}\left\{\phi_{j}(u, \lambda) \mathbf{f}(u, \lambda)\right\} d \lambda d u\right\} \\
& =O\left(\sqrt{T}\left(\frac{N^{2}}{T^{2}}+\frac{\log N}{N}+\frac{1}{T}\right)\right)=o(1) .
\end{aligned}
$$

\section{Testing Problem for Linear Dependence}

In this section we discuss the testing problem for linear dependence. The average measure of linear dependence is given by the following integral functional of time varying spectral density:

$$
\begin{aligned}
\lim _{T \rightarrow \infty} T^{-1} \sum_{t=1}^{T} M_{t, T}^{(X, Y)} & =\int_{0}^{1} \int_{-\pi}^{\pi}-\frac{1}{2 \pi} \log \left|I_{d^{(Y)}}-\mathbf{f}_{y x}(u, \lambda) \mathbf{f}_{x x}(u, \lambda)^{-1} \mathbf{f}_{x y}(u, \lambda) \mathbf{f}_{y y}(u, \lambda)^{-1}\right| d \lambda d u \\
& =\int_{0}^{1} \int_{-\pi}^{\pi} K_{(X, Y)}\{\mathbf{f}(u, \lambda)\} d \lambda d u,
\end{aligned}
$$


where

$$
K_{(X, Y)}\{\mathbf{f}(u, \lambda)\} \equiv-\frac{1}{2 \pi} \log \left|I_{d^{(Y)}}-\mathbf{f}_{y x}(u, \lambda) \mathbf{f}_{x x}(u, \lambda)^{-1} \mathbf{f}_{x y}(u, \lambda) \mathbf{f}_{y y}(u, \lambda)^{-1}\right| .
$$

We consider the testing problem for existence of linear dependence:

$$
H: \int_{0}^{1} \int_{-\pi}^{\pi} K_{(X, Y)}\{\mathbf{f}(u, \lambda)\} d \lambda d u=0
$$

against

$$
A: \int_{0}^{1} \int_{-\pi}^{\pi} K_{(X, Y)}\{\mathbf{f}(u, \lambda)\} d \lambda d u \neq 0
$$

For this testing problem, we define the test statistics $S_{T}$ as

$$
S_{T}=\sqrt{T} \int_{0}^{1} \int_{-\pi}^{\pi} K_{(X, Y)}\{\widehat{\mathbf{f}}(u, \lambda)\} d \lambda d u,
$$

then, we have the following result.

Theorem 4.1. Under $H$,

$$
S_{T} \stackrel{\oplus}{\longrightarrow} \mathcal{N}\left(0, V_{K_{(X, Y)}}^{2}\right)
$$

where the asymptotic variance of $S_{T}$ is given by

$$
\begin{aligned}
V_{K_{(X, Y)}}^{2}=4 \pi \int_{0}^{1}[ & \int_{-\pi}^{\pi} \operatorname{tr}\left[\mathbf{f}(u, \lambda) K_{(X, Y)}^{(1)}\{\mathbf{f}(u, \lambda)\}^{\prime}\right]^{2} d \lambda \\
& \left.+\frac{1}{4 \pi^{2}} \sum_{a, b, c, d} \kappa_{a, b, c, d} \gamma_{b, a}(u) \gamma_{c, d}(u)\right] d u,
\end{aligned}
$$

with

$$
\Gamma(u)=\{\gamma(u)\}_{a, b=1, \ldots, d^{(z)}}=\int_{-\pi}^{\pi} \mathbf{A}(u, \lambda)^{*} K_{(X, Y)}^{(1)}\{\mathbf{f}(u, \lambda)\} \mathbf{A}(u, \lambda) d \lambda,
$$

and $K_{(X, Y)}^{(1)}(\cdot)$ is the first derivative of $K_{(X, Y)}(\cdot)$. 
To simplify, $\left\{\mathbf{Z}_{t, T}\right\}$ is assumed to be Gaussian locally stationary process. Then, the asymptotic variance of $S_{T}$ becomes the integral functional of the time-varying spectral density:

$$
\begin{aligned}
V_{K_{(X, Y)}}^{2} & =4 \pi \int_{0}^{1} \int_{-\pi}^{\pi} \operatorname{tr}\left[\mathbf{f}(u, \lambda) K_{(X, Y)}^{(1)}\{\mathbf{f}(u, \lambda)\}^{\prime}\right]^{2} d \lambda \\
& =V_{K_{(X, Y)}}^{2}\{\mathbf{f}(u, \lambda)\} .
\end{aligned}
$$

If we take $\widehat{V}_{K_{(X, Y)}}^{2}=V_{K_{(X, Y)}}^{2}\{\widehat{\mathbf{f}}(u, \lambda)\}$, then $\widehat{V}_{K_{(X, Y)}}^{2}$ is consistent estimator of asymptotic variance, so, we have

$$
L_{T}=\frac{S_{T}}{\sqrt{\widehat{V}_{K_{(X, Y)}}^{2}}} \stackrel{\oplus}{\longrightarrow} \mathcal{N}(0,1) .
$$

Next, we introduce a measure of goodness of our test. Consider a sequence of alternative spectral density matrices:

$$
\mathbf{g}_{T}(u, \lambda)=\mathbf{f}(u, \lambda)+\frac{1}{\sqrt{T}} \mathbf{b}(u, \lambda)
$$

where $\mathbf{b}(u, \lambda)$ is a $d^{(Z)} \times d^{(Z)}$ matrix whose entries $b_{a b}(u, \lambda)$ are square-integrable functions on $[0,1] \times[-\pi, \pi]$.

Let $E_{\mathbf{g}_{T}}(\cdot)$ and $V_{\mathbf{f}}(\cdot)$ denote the expectation under $\mathbf{g}_{T}(u, \lambda)$ and the variance under $\mathbf{f}(u, \lambda)$, respectively. It is natural to define an efficacy of $L_{T}$ by

$$
\operatorname{eff}\left(L_{T}\right)=\lim _{T \rightarrow \infty} \frac{E_{\mathbf{g}_{T}}\left(S_{T}\right)}{\sqrt{V_{\mathbf{f}}\left(S_{T}\right)}}
$$

in line with the usual definition for a sequence of "parametric alternatives." Then we see that

$$
\begin{aligned}
\operatorname{eff}\left(L_{T}\right) & =\lim _{T \rightarrow \infty} \frac{\sqrt{T} \int_{-\pi}^{\pi}\left[K_{(X, Y)}\left\{\mathbf{g}_{T}(u, \lambda)\right\}-K_{(X, Y)}\{\mathbf{f}(u, \lambda)\}\right] d \lambda}{V_{K_{(X, Y)}}^{2}} \\
& =\frac{\int_{-\pi}^{\pi} \operatorname{tr}\left[K_{(X, Y)}^{(1)}\{\mathbf{f}(u, \lambda)\} \mathbf{b}(u, \lambda)^{\prime}\right] d \lambda}{V_{K_{(X, Y)}}^{2}} .
\end{aligned}
$$
to $L_{T}^{*}$ by

For another test $L_{T}^{*}$ we can define an asymptotic relative efficiency (ARE) of $L_{T}$ relative

$$
\operatorname{ARE}\left(L_{T}, L_{T}^{*}\right)=\left\{\frac{\operatorname{eff}\left(L_{T}\right)}{\operatorname{eff}\left(L_{\mathrm{T}}^{*}\right)}\right\}^{2} .
$$


Table 1: $L_{T}$ in (4.10) for each two companies.

\begin{tabular}{lccccccc}
\hline & $1: \mathrm{Hi}$ & $2: \mathrm{Ma}$ & $3: \mathrm{Sh}$ & $4: \mathrm{So}$ & $5: \mathrm{Ho}$ & $6: \mathrm{Ni}$ & 7:To \\
\hline 1:Hi & - & - & - & - & - & - & - \\
2:Ma & $\mathbf{1 8 . 7 9}$ & - & - & - & - & - & - \\
3:Sh & $\mathbf{1 9 . 8 6}$ & $\mathbf{1 8 . 9 3}$ & - & - & - & - & - \\
4:So & $\mathbf{1 9 . 2 2}$ & $\mathbf{1 9 . 1 8}$ & $\mathbf{1 8 . 2 7}$ & - & - & - & - \\
5:Ho & 15.35 & 14.46 & 15.17 & 15.42 & - & - & - \\
6:Ni & 15.18 & 15.03 & 15.84 & 16.58 & $\mathbf{1 9 . 2 4}$ & $\mathbf{-}$ & - \\
7:To & 15.86 & 16.06 & 16.00 & 16.61 & $\mathbf{2 0 . 5 7}$ & $\mathbf{1 9 . 1 2}$ & $\mathbf{-}$ \\
\hline
\end{tabular}

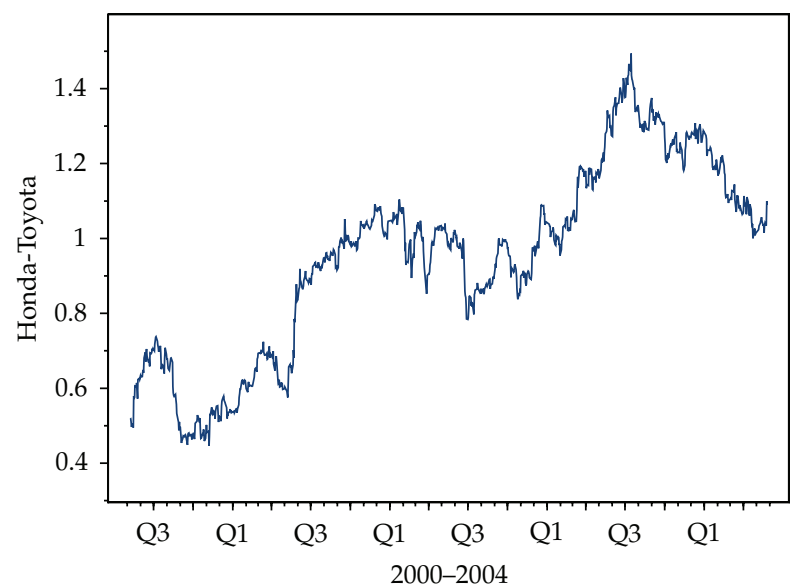

Figure 1: The daily linear dependence between HONDA and TOYOTA.

If we take the test statistic based on stationary assumption as another test $L_{T}^{*}$, we can measure the effect of nonstationarity when the process concerned is locally stationary process.

Finally, we discuss a testing problem of linear dependence for stock prices of Tokyo Stock Exchange. The data are daily log returns of 7 companies; 1 : HITACHI 2 : MATSUSHITA 3:SHARP 4:SONY 5:HONDA 6:NISSAN 7: TOYOTA. The individual time series are 1174 data points since December 28, 1999 until October 1, 2004. We compute $L_{T}$ in (4.10) for each two companies. The selected parameters are $T=1000, N=175$, and $M=8$, where $N$ is the length of segment which the localized periodogram is taken over and $M$ is the bandwidth of the weight function.

The results are listed in Table 1. It shows that all values for each two companies are large. Since under null hypothesis the limit distribution of $L_{T}$ is standard normal, we can conclude hypothesis is rejected. Namely, the linear dependencies exist at each two companies. In particular, the values both among electric appliance companies and among automobile companies are significantly large. Therefore, we can see that the companies in the same business have strong dependence.

In Figures 1 and 2, the daily linear dependence between HONDA and TOYOTA and between HITACHI and SHARP is plotted. They show that the daily dependencies are not 


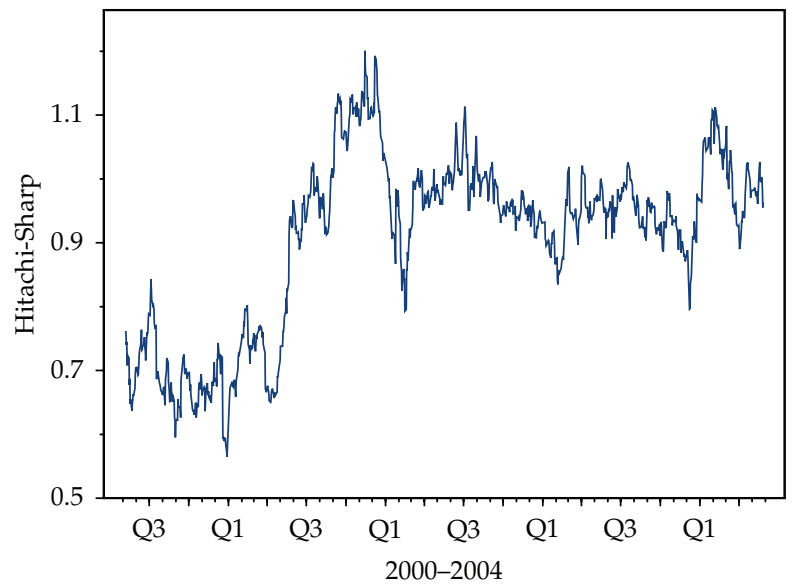

Figure 2: The daily linear dependence between HITACHI and SHARP.

constant and change in time. So, it seems to be reasonable that we use the test statistic based on nonstationary assumption.

\section{References}

[1] J. Geweke, "Measurement of linear dependence and feedback between multiple time series," Journal of the American Statistical Association, vol. 77, no. 378, pp. 304-313, 1982.

[2] Y. Hosoya, "The decomposition and measurement of the interdependency between second-order stationary processes," Probability Theory and Related Fields, vol. 88, no. 4, pp. 429-444, 1991.

[3] M. Taniguchi, M. L. Puri, and M. Kondo, "Nonparametric approach for non-Gaussian vector stationary processes," Journal of Multivariate Analysis, vol. 56, no. 2, pp. 259-283, 1996.

[4] M. B. Priestley, Spectral Analysis and Time Series, Academic Press, London, UK, 1981.

[5] R. Dahlhaus, "On the Kullback-Leibler information divergence of locally stationary processes," Stochastic Processes and Their Applications, vol. 62, no. 1, pp. 139-168, 1996.

[6] R. Dahlhaus, "Maximum likelihood estimation and model selection for locally stationary processes," Journal of Nonparametric Statistics, vol. 6, no. 2-3, pp. 171-191, 1996.

[7] R. Dahlhaus, "Fitting time series models to nonstationary processes," The Annals of Statistics, vol. 25, no. 1, pp. 1-37, 1997.

[8] R. Dahlhaus, "A likelihood approximation for locally stationary processes," The Annals of Statistics, vol. 28, no. 6, pp. 1762-1794, 2000.

[9] Yu. A. Rozanov, Stationary Random Processes, Holden-Day, San Francisco, Calif, USA, 1967.

[10] R. Dahlhaus, "Asymptotic statistical inference for nonstationary processes with evolutionary spectra," in Athens Conference on Applied Probability and Time Series 2, vol. 115 of Lecture Notes in Statist, pp. 145-159, Springer, New York, NY, USA, 1996.

[11] K. Sakiyama and M. Taniguchi, "Discriminant analysis for locally stationary processes," Journal of Multivariate Analysis, vol. 90, no. 2, pp. 282-300, 2004. 


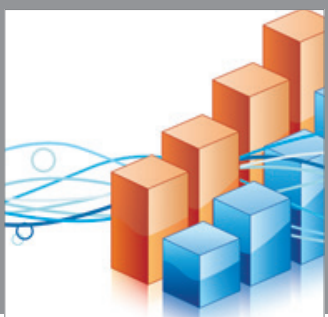

Advances in

Operations Research

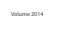

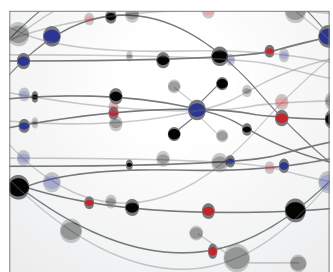

\section{The Scientific} World Journal
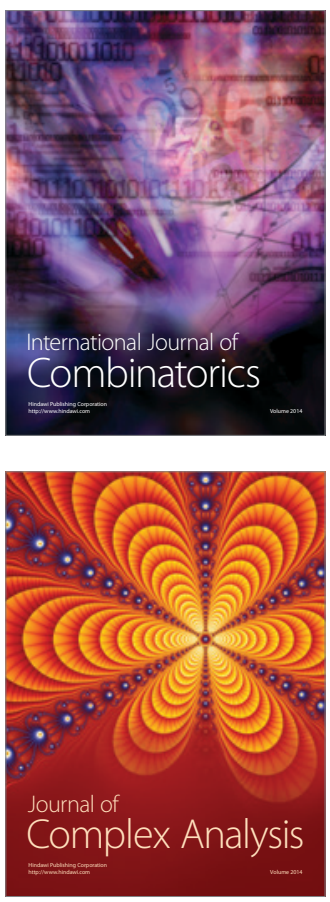

International Journal of

Mathematics and

Mathematical

Sciences
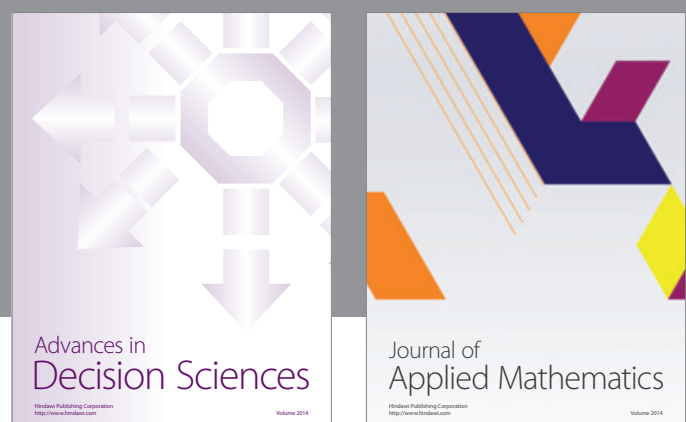

Journal of

Applied Mathematics
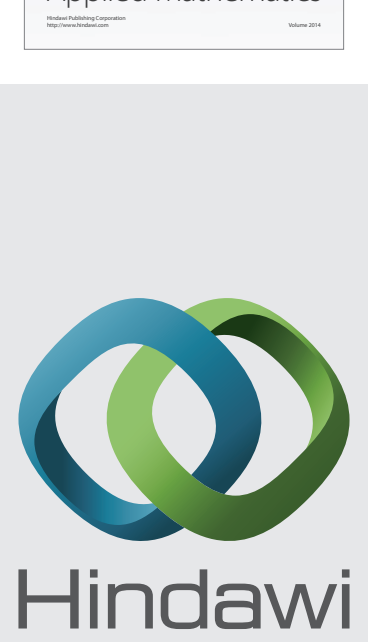

Submit your manuscripts at http://www.hindawi.com
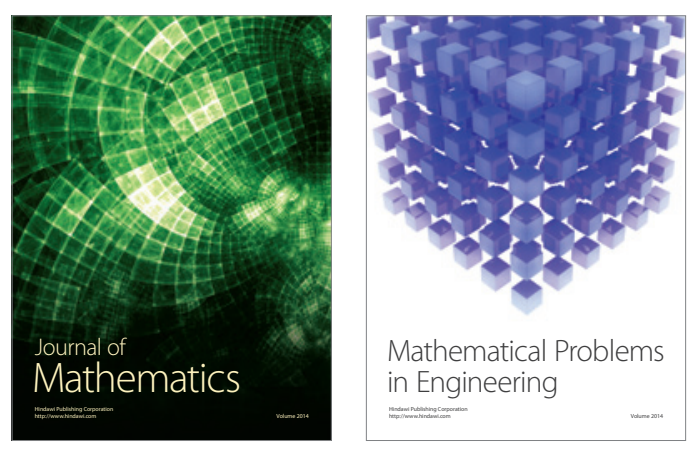

Mathematical Problems in Engineering
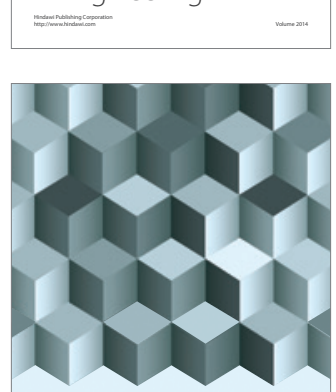

Journal of

Function Spaces
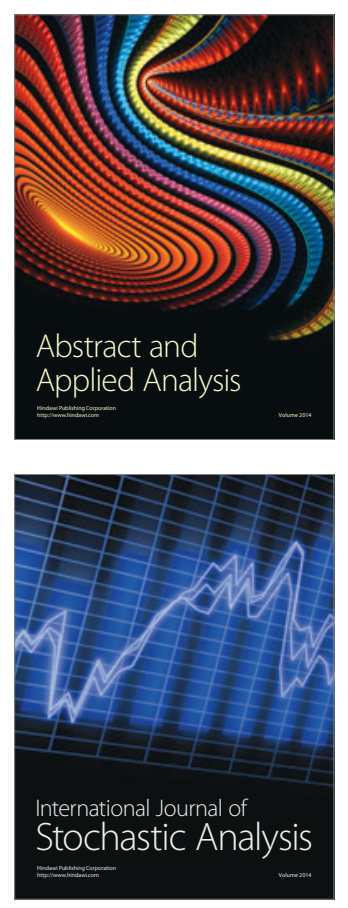

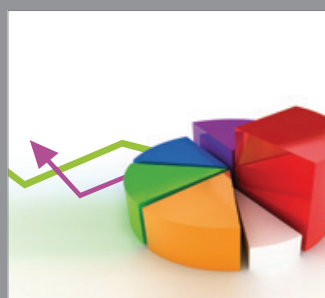

ournal of

Probability and Statistics

Promensencen
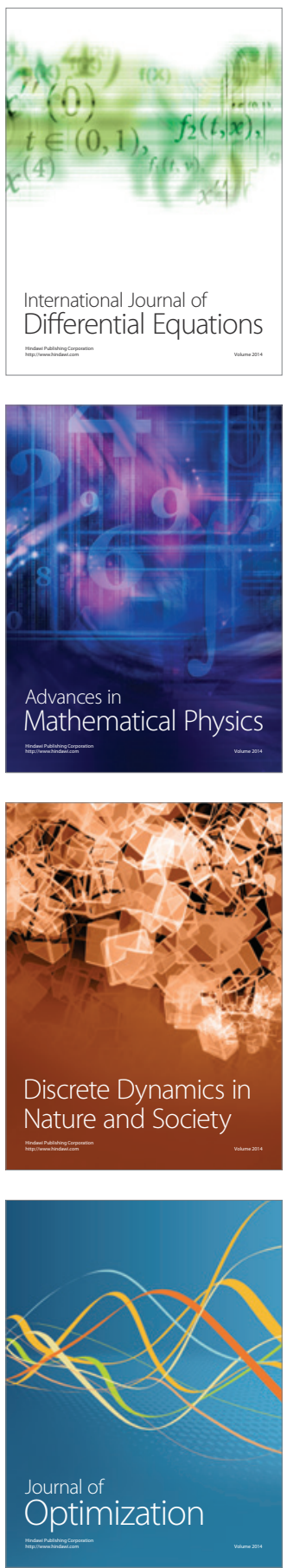